\title{
The Fermi-LAT and H.E.S.S. views of the supernova remnant W49B
}

Francois Brun*

DSM/Irfu, CEA Saclay, Gif-sur-Yvette, France

E-mail: francois.brunecea.fr

\section{Marianne Lemoine-Goumard}

Centre d'Études Nucléaires de Bordeaux Gradignan, Gradignan, France

E-mail: lemoinedcenbg.in2p3. fr

\section{Vincent Marandon}

Max-Planck-Institut für Kernphysik, Heidelberg, Germany.

E-mail: marandon@mpi-hd.mpg.de

\section{Junichiro Katsuta}

Hiroshima University, Hiroshima, Japan

E-mail: katsuta@hep01.hepl.hiroshima-u.ac.jp

\section{Tobias Jogler}

SLAC National Accelerator Laboratory, 2575 Sand Hill Road M/S 29, Menlo Park, CA 94025,

$U S A$

E-mail: jogler@slac.stanford.edu

\section{on behalf of the H.E.S.S. and Fermi-LAT Collaborations}

The supernova remnant (SNR) W49B is a mixed-morphology remnant interacting with molecular clouds (MC) which originated in a Type Ib/Ic supernova explosion that occurred between one to four thousand years ago. It is one of the brightest SNRs in our Galaxy as seen in radio and Xrays. Gamma-ray observations of SNR/MC are a powerful tool to constrain the origin of Galactic cosmic rays, as they can probe the acceleration of hadrons through their interaction with the surrounding medium and subsequent emission of high-energy photons. W49B has been detected in gamma-rays at high energies $(0.1-100 \mathrm{GeV})$ and very high energies $(>100 \mathrm{GeV})$ with the FermiLAT and the H.E.S.S. Cherenkov telescopes, respectively. The latest results obtained on W49B with these instruments will be presented. In particular, we will report on the detection of a spectral break at low energies with the Fermi-LAT, similar to that previously found in other SNRs and interpreted as the signature of gamma-ray emission produced through neutral-pion decay. The implications of these results on the particle population and the physical processes leading to the observed gamma-ray emission in W49B will be discussed.

The 34th International Cosmic Ray Conference,

30 July- 6 August, 2015

The Hague, The Netherlands

${ }^{*}$ Speaker. 
Further details will be available in an upcoming publication by the H.E.S.S. and Fermi-LAT Collaborations. 